\section{A $70 \%$ cut-off for MYC protein expression in diffuse large B-cell lymphoma identifies a high-risk group of patients}

We recently examined the reproducibility of MYC and BCL-2 immunohistochemical (IHC) scoring and the impact of high expression of MYC and BCL-2 (double expresser status, DE) on survival and progression in a large retrospective cohort of aggressive B-cell lymphoma patients treated with rituximab plus cyclophosphamide, doxorubicin, vincristine and prednisone (R-CHOP) or R-CHOP-like regimens. ${ }^{1}$ We found that IHC scoring for MYC and BCL-2 was highly reproducible when cut-off values of $\geq 70 \%$ for MYC and $\geq 50 \%$ for BCL- 2 were used. This threshold also predicted the presence of gene rearrangements identifying MYC translocations in $88 \%$ of cases. Patients with dual MYC expression of $\geq 70 \%$ and BCL- 2 expression of $\geq 50 \%$ showed a significantly inferior clinical course and, therefore, represent candidates for novel treatment modalities. ${ }^{1}$ We have now validated these findings in an independent cohort of 461 patients enrolled in prospective clinical trials of the German High-Grade Non-Hodgkin Lymphoma Study Group (DSHNHL).,3

In these trials, patients underwent R-CHOP-14 if $>60$ years of age and R-CHOEP/R-MegaCHOEP if $\leq 60$ years of age. In the MegaCHOEP trial reported by Schmitz et al., ${ }^{4}$ no significant differences in outcome between RCHOEP-14 and R-MegaCHOEP had been observed, but to date, no randomized trial has been conducted to answer if R-CHOEP in younger patients is superior in comparison with R-CHOP. In a subgroup analysis for young low-risk patients from the MInT trial reported by Pfreundschuh et al., ${ }^{5}$ no difference in outcome was observed between R-CHOEP-21 and R-CHOP-21. In elderly patients, the Cunningham trial ${ }^{6}$ revealed that the outcome of R-CHOP-14 is not better than that of $\mathrm{R}-\mathrm{CHOP}-21$. In the German cohort of 428 patients with MYC and BCL-2 IHC scoring available, 104 cases (24\%) were $\mathrm{MYC}^{-} / \mathrm{BCL}^{-}{ }^{-}$(double negative, DN), $283(66 \%)$ were $\mathrm{MYC}^{-} / \mathrm{BCL}^{+} 2^{+}$(BCL2only), 8 (2\%) were $\mathrm{MYC}^{+} / \mathrm{BCL}^{-} 2^{-}$(MYConly), and 33 were $\mathrm{MYC}^{+} / \mathrm{BCL}-2^{+}$ using the above-mentioned cut-off values, meaning that $8 \%$ of DLBCL were assigned a DE status. Results from both MYC IHC scoring and MYC fluorescence in situ hybridization (FISH) were available from samples of 415 patients. In this analysis, 19 of $43(44 \%)$ samples with high MYC expression (70/71-100\%) harbored a MYC translocation (Table 1). The lower number of cases noted in our report with both high MYC expression and MYC breakage in comparison with the Ambrosio paper ${ }^{1}$ are not easily explained. Most probably, this is due to a difference in the genetic constitution of the two different patient populations that were examined or to the analysis strategy: in the German cohort, the analysis was made on TMA while in the paper of Ambrosio et al. ${ }^{1}$ full sections were analyzed. ${ }^{7}$ According to the results of molecular cell of origin (COO) analysis, we identified $50 \%$ of patients with an $\mathrm{ABC}$ subtype within the DE cohort using a MYC cut-off point of $70 \%$ and $68 \%$ using a cut-off point of $40 \%$. The sample sizes, however, are too small to conclude that the groups differ from the proportion of the $\mathrm{ABC}$ subtype. It has to be stressed, however, that the DE status does not identify a homogeneous biological group of tumors and, especially, that the DE status in ABCDLBCL arises through very different mechanisms.

In the German cohort, the DE subgroup had a significant inferior clinical course, while the DN subset had a
Table 1. Results from both MYC immunohistochemical (IHC) scoring and MYC fluorescence in situ hybridization.

\begin{tabular}{lccc} 
MYC IFC & \multicolumn{2}{c}{ MYC break } & \\
\cline { 2 - 3 } & Negative & Positive & Total \\
$0-40 \% *$ & $257(97 \%)$ & $7(3 \%)$ & $264(64 \%)$ \\
$40 \%-70 \% *$ & $96(89 \%)$ & $12(11 \%)$ & $108(26 \%)$ \\
\hline $70 \%-100 \% *$ & $24(56 \%)$ & $19(44 \%)$ & $43(10 \%)$ \\
Total & $377(91 \%)$ & $38(9 \%)$ & $415(100 \%)$ \\
\hline
\end{tabular}

$P<0.001$. *Cut-off points were slightly different between clinical trials included in the analysis.

superior outcome and the $\mathrm{MYC}^{-} / \mathrm{BCL}^{-} 2^{+}$subset had an intermediate prognosis. The differences were statistically significant for event-free survival (EFS), progression-free survival (PFS), and overall survival (OS) (EFS: DN vs. DE, $P<0.001$; DN vs. BCL2only $P=0.004$; BCL2only vs. DE $P=0.032$ ) (Figure 1A-C). These results could be confirmed in a multivariate analysis (Hazard ratios [HR] for DE vs. other: EFS: 2.1 95\%CI:1.2-3.5, $P=0.005$; PFS: 2.5 95\%CI:1.5-4.3, $P=0.001$; OS: 2.7 95\%CI:1.5-4.8, $P=0.001)$ adjusted for the factors of the International Prognostic Index (IPI) (age $>60$ years, lactate dehydrogenase $[\mathrm{LDH}]>\mathrm{N}$, Eastern Cooperative Oncology Group [ECOG] $>1$, stage III/IV, extralymphatic involvement $>1$ ). In multivariate analyses adjusted for the International Prognostic Index (IPI) factors (age $>60$ years, LDH $>N$, ECOG $>1$, stage III/IV and more than one site of extralymphatic involvement) both MYC (70/71-100\% vs. other) and BCL2 (50/51-100\% vs. other) expression were significant risk factors in EFS (MYC: HR1.9, 95\%CI: 1.2-3.1, $P=0.007$ and BCL2: HR1.8, 95\%CI: 1.2-2.7, $P=0.006$ ), PFS (MYC: HR2.1, 95\%CI: $1.3-3.5, P=0.004$ and BCL2: HR2.4, 95\%CI: 1.5-3.8, $P<0.001)$ and OS (MYC: HR2.3, 95\%CI: 1.3-4.0, $P=0.004$ and BCL2: HR2.0, 95\%CI: $1.2-3.3$ and $P=0.009$ ). When cases were stratified according to MYC protein expression only, patients with $M Y C \geq 70 \%$, again, experienced inferior outcome in EFS $(P=0.005)$, PFS $(P=0.004)$, and OS $(P=0.002)$ in comparison with patients with low MYC expression $(\leq 40 \%)$ (Figure 1D-F), while no difference in prognosis was seen between patients whose tumors had MYC expression $\leq 40 \%$ and $>40-70 \%$. Within the DE group, the occurrence of a genetic double hit for $M Y C$ and $B C L-2(\mathrm{n}=8$ of $32,25 \%)$ failed to confer a significant prognostic difference in EFS $(P=0.628)$, PFS $(P=0.375)$, and OS $(P=0.059)$ between patients with $\mathrm{DH}$ positive and $\mathrm{DH}$ negative tumors (Figure $2 \mathrm{~A}-\mathrm{C}$ ). Within the nonDE group, we observed a genetic double hit for MYC and BCL-2 in only 11 of 354 (3\%) patients with no relevant survival differences between patients with $\mathrm{DH}$ positive and DH negative tumors (Figure 2D-F). However, due to the low number of events, these results have to be interpreted with caution.

In essence, these results are in agreement with our previous findings indicating that high $(\geq 70 \%)$ MYC expression identifies a subset of DLBCL with adverse clinical outcome independent of the presence of a double hit of $M Y C$ and $B C L-2$.

Increasing evidence suggests that the sole identification of the double hit (MYC and BCL-2) status may not be the optimal tool to identify patients in need of alternative therapies and in many studies, a proportion of DE patients nevertheless experience long-term survival. Two recent papers shed light on this seeming discrepancy. ${ }^{8,9}$ In the first paper, the authors defined a clinically and biologically distinct subgroup of aggressive lymphomas with 
EFS

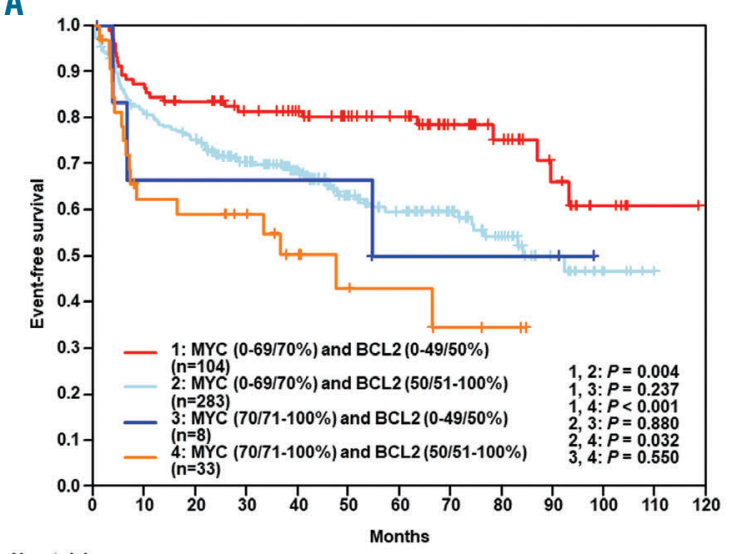

No. at risk

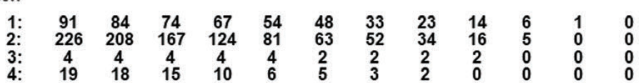

C

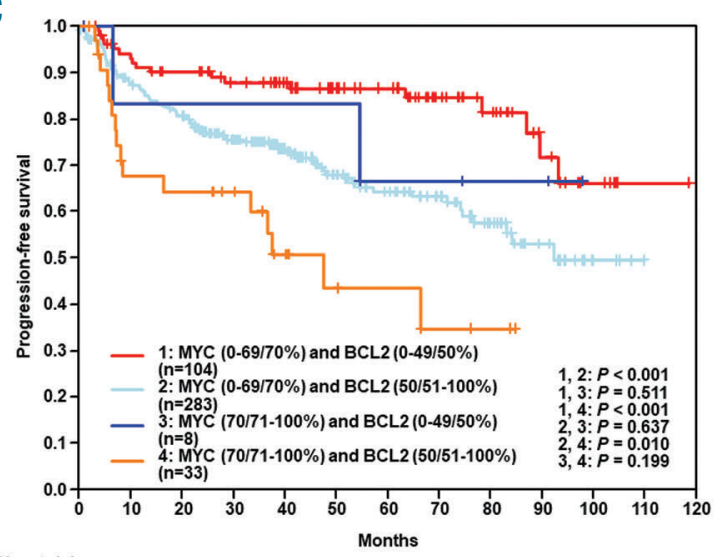

No. at risk

$\begin{array}{ccccccccccccc}1: & 94 & 87 & 77 & 69 & 56 & 50 & 35 & 24 & 14 & 6 & 1 & 0 \\ 2 & 237 & 216 & 174 & 128 & 84 & 66 & 52 & 34 & 16 & 5 & 0 & 0 \\ 3: & 50 & 5 & 5 & 5 & 5 & 3 & 3 & 2 & 2 & 2 & 0 \\ 4: & 20 & 19 & 16 & 10 & 6 & 5 & 3 & 2 & 0 & 0 & 0 & 0\end{array}$

E

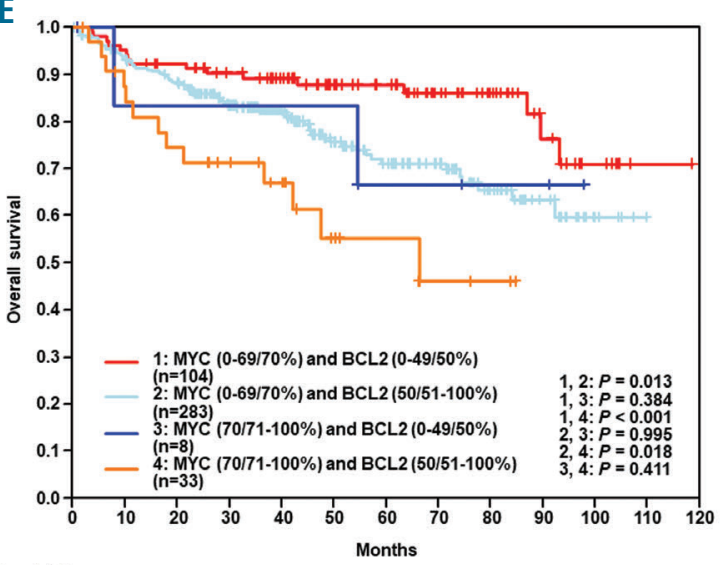

No. at risk

$$
\begin{array}{lllllllllllll}
1: & 99 & 93 & 84 & 75 & 60 & 53 & 39 & 27 & 15 & 7 & 1 & 0 \\
2 & 258 & 242 & 197 & 144 & 94 & 75 & 60 & 40 & 20 & 6 & 0 & 0 \\
3: & 57 & 53 & 59 & 5 & 5 & 3 & 3 & 2 & 20 & 0 & 0 & 0 \\
4 & 27 & 23 & 19 & 15 & 8 & 6 & 3 & 2 & 0 & 0 & 0 & 0
\end{array}
$$

B

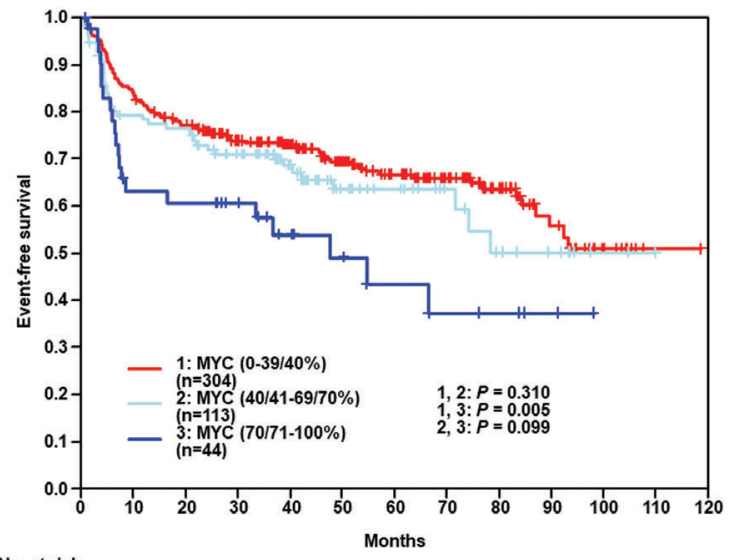

No. at risk

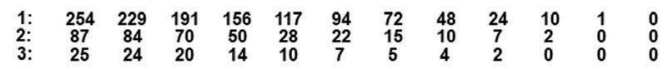

PFS

D

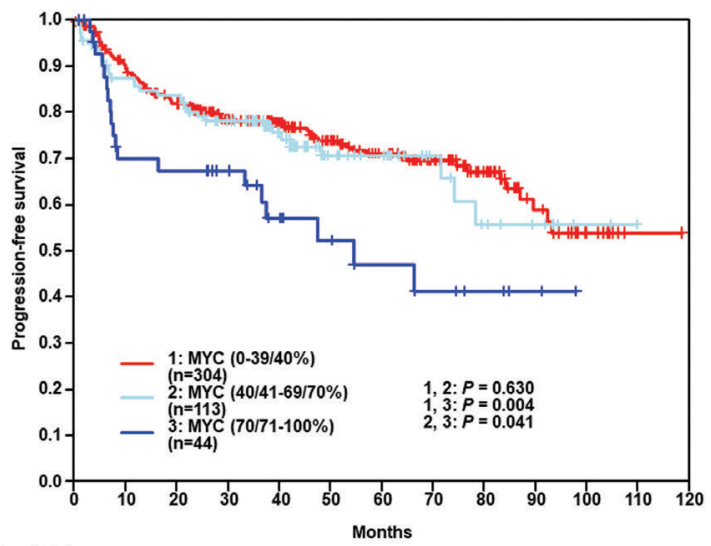

No. at risk

$\begin{array}{ccccccccccccc}\text { 1: } & 262 & 234 & 195 & 159 & 120 & 97 & 74 & 49 & 24 & 10 & 1 & 0 \\ 2: & 95 & 91 & 77 & 54 & 30 & 24 & 15 & 10 & 7 & 2 & 0 & 0 \\ 3: & 27 & 26 & 22 & 15 & 11 & 8 & 6 & 4 & 2 & 0 & 0 & 0\end{array}$

OS

$F$

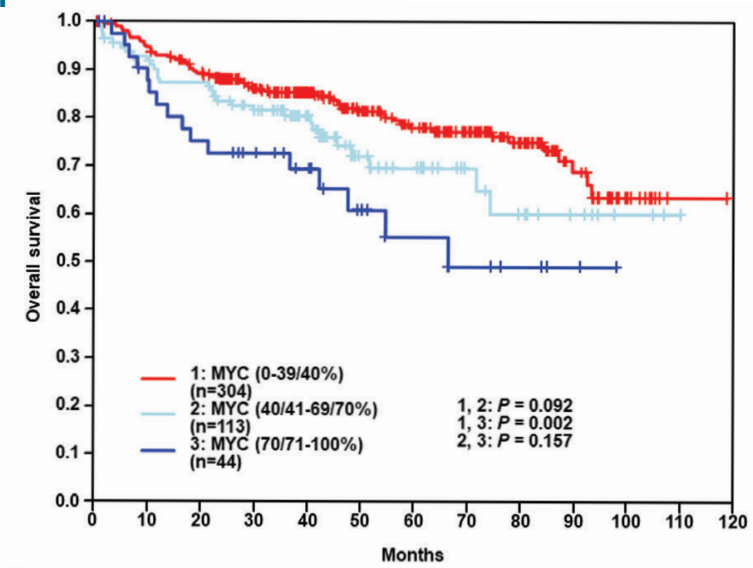

No. at risk

$\begin{array}{ccccccccccccc}1: & 285 & 264 & 223 & 178 & 134 & 109 & 86 & 57 & 29 & 11 & 1 & 0 \\ 2: & 101 & 95 & 80 & 58 & 31 & 24 & 15 & 11 & 7 & 3 & 0 & 0 \\ 3: & 35 & 30 & 25 & 20 & 13 & 9 & 6 & 4 & 2 & 0 & 0 & 0\end{array}$

Figure 1. Event-free survival (EFS), progression-free survival (PFS) and overall survival (OS) of patients stratified according to Myc and BCL-2 expression. (A-C) The double expressor (DE) subgroup had a significant inferior clinical course, while the double negative (DN) subset had a superior outcome and the MYC-/BCL$2^{+}$subset had an intermediate prognosis, with the differences being statistically significant for EFS, PFS and OS. (D-F) The cases were stratified according to MYC protein expression only, patients with MYC $>70 \%$ experienced inferior outcome in EFS $(P=0.005)$, PFS $(P=0.004)$ and OS $(P=0.002)$ in comparison to patients with low MYC expression (<40\%). No difference in prognosis was seen between patients whose tumors had MYC expression $<40 \%$ and $40-70 \%$. 


\section{EFS}

A

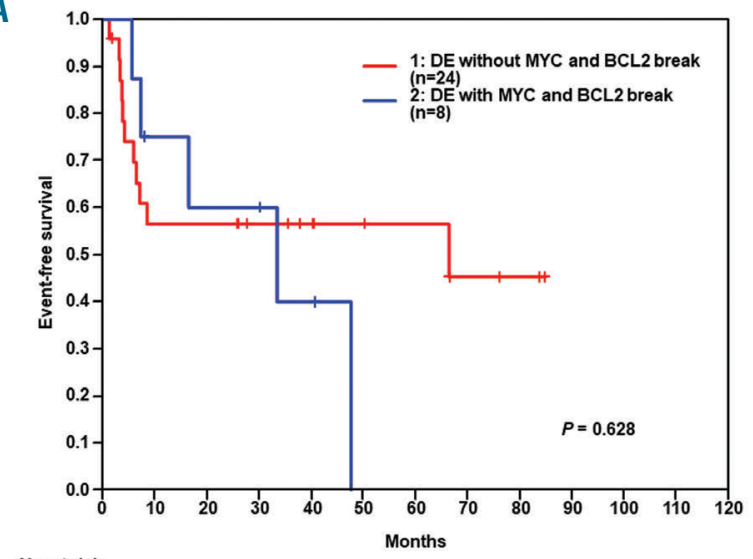

No. at risk

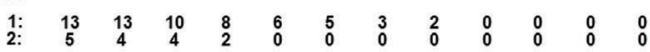

C

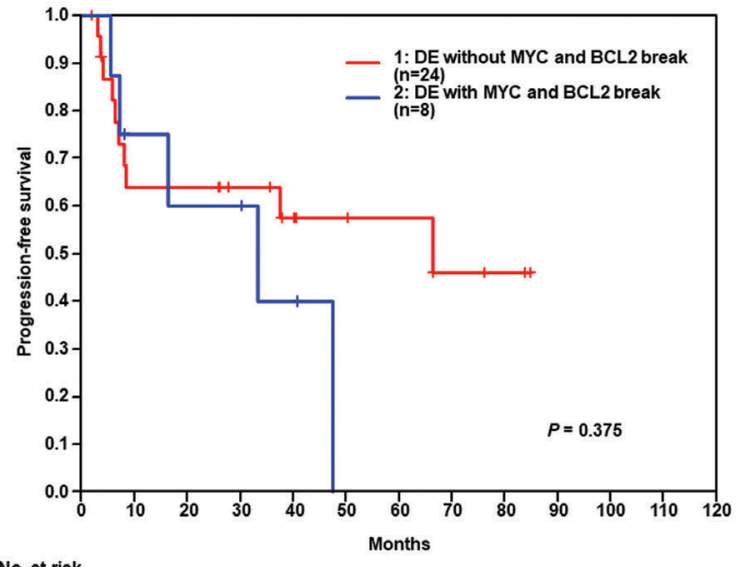

No. at risk

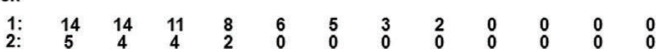

\section{E}

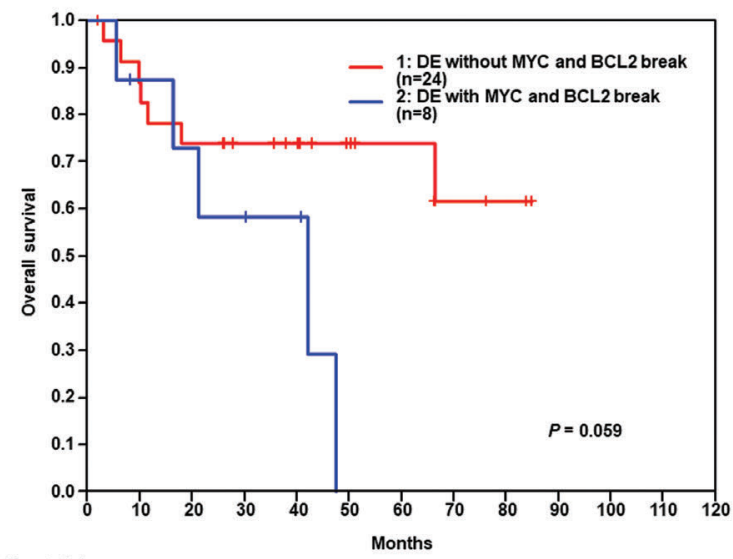

No. at risk

$\begin{array}{ccccccccccccc}1: & 20 & 17 & 14 & 12 & 8 & 6 & 3 & 2 & 0 & 0 & 0 & 0 \\ 2 & 6 & 4 & 4 & 0 & 0 & 0 & 0 & 0 & 0 & 0 & 0\end{array}$
B

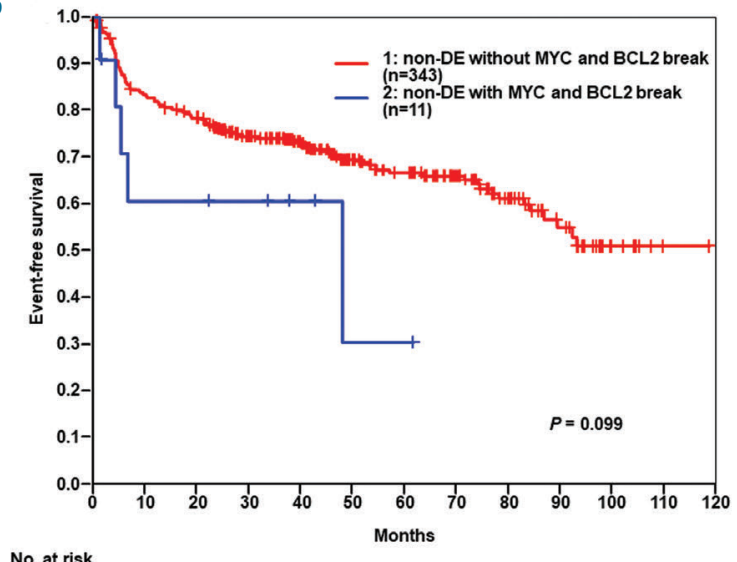

No. at risk

$\begin{array}{cccccccccccccc}1: & 282 & 262 & 217 & 177 & 129 & 106 & 82 & 56 & 30 & 9 & 1 & 0 \\ 6 & 6 & 6 & 1 & 1 & 0 & 0 & 0 & 0 & 0 & 0\end{array}$

PFS

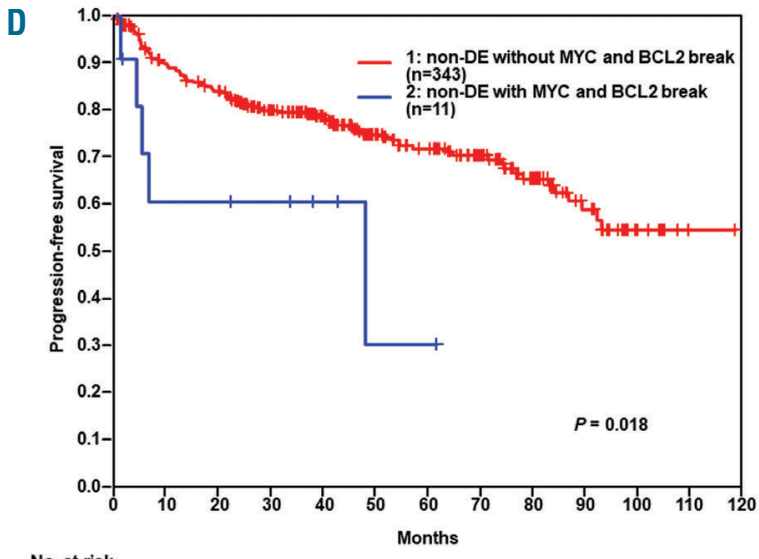

No. at risk

$\begin{array}{ccccccccccccc}1: & 294 & 272 & 227 & 184 & 135 & 112 & 85 & 57 & 30 & 9 & 1 & 0\end{array}$

OS

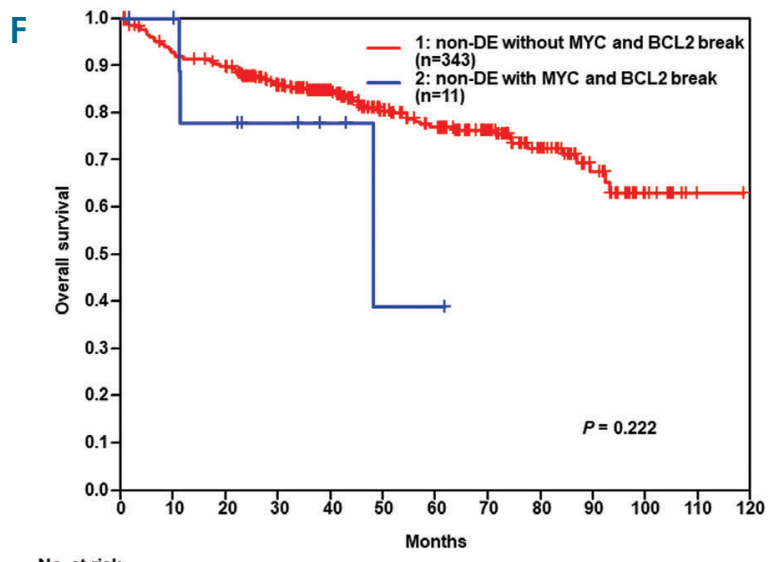

No. at risk

$\begin{array}{lllllllllllll}\text { 1: } & 313 & 300 & 256 & 204 & 149 & 124 & 97 & 65 & 35 & 11 & 1 & 0 \\ 2 & 10 & 7 & 5 & 1 & 1 & 0 & 0 & 0 & 0 & 0 & 0\end{array}$

Figure 2. Comparison of double hit in double expressor and non-double expressor (DE). (A-C) Within the DE group, the occurrence of a genetic double hit for MYC and BCL-2 ( $n=8$ of 32, 25\%) failed to confer a significant prognostic difference in EFS $(P=0.628)$, PFS $(P=0.375)$ and OS $(P=0.059)$ between patients with DH positive and DH negative tumors. (D-F) Within the non DE group, the occurrence of a genetic double hit for MYC and BCL-2 ( $n=11$ of 354 , $3 \%$ ) failed to confer a significant prognostic difference in EFS $(P=0.099)$ and OS $(P=0.222)$ between patients with DH positive and DH negative tumors. 
inferior prognosis among GCB-DLBCL. This tumor subgroup was characterized by a gene expression signature derived from HGBL-DH/TH lymphomas (DHIT signature).$^{8}$ Using this signature, however, only $50 \%$ of the cases stratified into the subgroup actually had dual rearrangements of $M Y C$ and $B C L-2$ genes, and some DE cases were not assigned into the DHIT signature positive group. Gene set enrichment analysis demonstrated (over-) expression of MYC and E2F target genes, and of genes associated with oxidative phosphorylation and MTORC1 signaling in the DHIT-positive tumors, implying a pivotal role for MYC protein expression irrespective of the DH status. Unfortunately, the study did not document the precise percentage of MYC protein expression; it also did not correlate MYC protein expression to $M Y C$ gene rearrangements. The second paper identified $9 \%$ of DLBCL (83 of 928) as "molecular high grade (MHG)" Bcell lymphomas using gene expression analysis. ${ }^{9}$ Most MHG (75 of 83) were GCB-like, and again, only half of them were $M Y C$ rearranged or double-hit lymphomas. The MHG subset treated with R-CHOP had a significantly poorer outcome than MHG negative DLBCL. Furthermore, in vivo experiments demonstrated that MYC-expressing lymphoma cells were obviously addicted to its oncogenic effect and, therefore, were critically relying on MYC expression regardless of MYC gene rearrangements. ${ }^{10}$

Although genomic testing has entered clinical practice, sophisticated tests like those reported are not yet widely available in all laboratories. Therefore, gene expression signatures identifying high-risk subgroups are currently difficult to apply in the clinic. Our findings describe a more readily available tool to identify patients at risk with a high MYC protein expression cut-off circumventing problems related to interobserver variability. ${ }^{11}$ Our findings are corroborated in a recent paper by Pedersen $e t$ al. ${ }^{12}$ who demonstrated that stratification by MYC expression has prognostic impact in MYC translocated DLBCL. ${ }^{12}$

In summary, we have confirmed that the prognosis of DLBCL is inversely correlated with MYC protein expression levels, and, by using diagnostic thresholds of high reproducibility, we were able to identify a subset of patients with adverse outcome in need of alternative therapeutic strategies.

Marita Ziepert, ${ }^{1}$ Stefano Lazzi, ${ }^{2}$ Raffaella Santi, Federica Vergoni, ${ }^{3}$ Massimo Granai, ${ }^{2}$ Virginia Mancini, ${ }^{2}$ Annette M. Staiger, ${ }^{4,5}$ Heike Horn, ${ }^{4,5}$ Markus Löffler, ${ }^{6}$ Viola Pöschel, ${ }^{7}$ Gerhald Held, ${ }^{7}$ Gerald Wulf, Lorenz H. Trümper, Norbert Schmitz, ${ }^{10}$ Andreas Rosenwald, ${ }^{11}$ Elena Sabattini, ${ }^{12}$ Kikkeri N. Naresh, ${ }^{13}$ Harald Stein, ${ }^{14}$ German Ott and Lorenzo Leoncini ${ }^{2}$

${ }^{1}$ University of Leipzig - Institute of Medical Informatics, Statistics and Epidemiology, Leipzig, Germany; '2Department of Medical Biotechnology, Section of Pathology, University of Siena, Siena, Italy; ${ }^{3}$ Department of Pathology, Careggi University Hospital, University of Firenze, Firenze, Italy; ${ }^{4}$ Department of Clinical Pathology, RobertBosch-Krankenhaus, Stuttgart, Germany; ${ }^{5}$ Dr. Margarete FischerBosch-Institute of Clinical Pharmacology, Stuttgart, Germany and University of Tübingen, Germany; ${ }^{6}$ Institute for Medical Informatics,
Statistics and Epidemiology, University of Leipzig, Leipzig, Germany; ${ }^{7}$ DSHNHL Studiensekretariat, Universitätsklinikum des Saarlandes,

Homburg, Germany; Department of Hematology and Oncology, Georg-August Universität, Göttingen, Germany; ${ }^{9} \mathrm{G}$-CCC

(Göttingen Comprehensive Cancer Center), University Medicine Göttingen, Göttingen, Germany; ${ }^{10}$ Department of Medicine A, University Hospital Münster, Münster, Germany; "Institute of Pathology, Universität Würzburg and Comprehensive Cancer Center Mainfranken (CCCMF), Würzburg, Germany; ${ }^{12}$ Institute of Hematology "L. and A. Seràgnoli", S. Orsola - Malpighi Hospital, Bologna, Italy; ${ }^{13}$ Hammersmith Hospital and Imperial College,

London, UK; ${ }^{14}$ Pathodiagnostik Berlin, Berlin, Germany and

${ }^{15}$ Department of Clinical Pathology, Robert-Bosch-Krankenhaus,

Stuttgart, Germany

Correspondence:

LORENZOLEONCINI - lorenzo.leoncini@dbm.unisi.it

doi:10.3324/haematol.2019.235556

\section{References}

1. Ambrosio MR, Lazzi S, Lo Bello G, et al. MYC protein expression scoring and its impact on the prognosis of aggressive B-cell lymphoma patients. Haematologica. 2019;104(1):e25-e28

2. Staiger AM, Altenbuchinger M, Ziepert, et al. A novel lymphomaassociated macrophage interaction signature (LAMIS) provides robust risk prognostication in diffuse large B-cell lymphoma clinical trial cohorts of the DSHNHL. Leukemia. 2019;34(2):543-552.

3. Mellert K, Martin M, Lennerz JK, et al. The impact of SOCS1 mutations in diffuse large B-cell lymphoma. Br J Haematol. 2019;187(5):627-637

4. Schmitz N, Nickelsen M, Ziepert M, et al. Conventional chemotherapy (CHOEP-14) with rituximab or high-dose chemotherapy (MegaCHOEP) with rituximab for young, high-risk patients with aggressive B-cell lymphoma: an open-label, randomised, phase 3 trial (DSHNHL 2002-1). Lancet Oncol. 2012;13(12):1250-1259

5. Pfreundschuh M, Trümper L, Osterborg A, et al. CHOP-like chemotherapy plus rituximab versus CHOP-like chemotherapy alone in young patients with good-prognosis diffuse large-B-cell lymphoma: a randomised controlled trial by the MabThera International Trial (MInT) Group. Lancet Oncol. 2006;7(5):379-391.

6. Cunningham D, Hawkes EA, Jack A, et al. Rituximab plus cyclophosphamide, doxorubicin, vincristine, and prednisolone in patients with newly diagnosed diffuse large B-cell non-Hodgkin lymphoma: a phase 3 comparison of dose intensification with 14-day versus 21-day cycles. Lancet. 2013;25;381(9880):1817-1826.

7. Hupp M, Williams S, Dunnette B, et al. Comparison of evaluation techniques, including digital image analysis, for MYC protein expression by immunohistochemical stain in aggressive B-cell lymphomas. Hum Pathol. 2019;83:124-132.

8. Ennishi D, Jiang A, Boyle M, et al. Double-hit gene expression signature defines a distinct subgroup of germinal center B-cell-like diffuse large B-cell lymphoma. J Clin Oncol. 2019;37(3):190-201.

9. Sha C, Barrans S, Cucco F, et al. Molecular high-grade B-cell lymphoma: defining a poor-risk group that requires different approaches to therapy. J Clin Oncol. 2019;37(3):202-212

10. Li W, Gupta SK, Han W, et al. Targeting MYC activity in double-hit lymphoma with MYC and BCL2 and/or BCL6 rearrangements with epigenetic bromodomain inhibitors. J Hematol Oncol. 2019;12(1):73.

11. Mahmoud AZ, George TI, Czuchlewski DR, et al. Scoring of MYC protein expression in diffuse large B-cell lymphomas: concordance rate among hematopathologists. Mod Pathol. 2015;28(4):545-551.

12. Pedersen $M \varnothing$, Gang $A O$, Clasen-Linde E, et al. Stratification by MYC expression has prognostic impact in MYC translocated B-cell lymphoma identifies a subgroup of patients with poor outcome. Eur J Haematol. 2019;102(5):395-406. 